\title{
Allergic reactions due to concomitant administration of multiple drugs in intravenous fluid in emergency departments in Turkey
}

\author{
Mehmet Erdem Çakmak, ${ }^{1}$ Saltuk Buğra Kaya, ${ }^{2}$ Özge Can Bostan, ${ }^{3}$ Ebru Damadoğlu, ${ }^{3}$ \\ Gül Karakaya, ${ }^{3}$ Ali Fuat Kalyoncu ${ }^{3}$
}

${ }^{1}$ Clinic of Allergy and Clinical Immunology, Başakşehir Çam ve Sakura City Hospital, Istanbul; ${ }^{2}$ Clinic of Allergy and Clinical Immunology, Erzurum Training and Research Hospital, Erzurum; ${ }^{3}$ Department of Chest Diseases, Division of Allergy and Clinical Immunology, Hacettepe University School of Medicine, Ankara, Turkey

\section{Abstract \\ The present study aimed to evaluate patients who were referred}

Correspondence: Mehmet Erdem Çakmak, Clinic of Allergy and Clinical Immunology, Başakşehir Çam ve Sakura City Hospital, G-434 Street No: 2L, 34480, Başakşehir, Istanbul, Turkey.

Tel.: +90.0505.4869750; Fax: +90.312.3100809

E-mail: mehmeterdemcakmak@gmail.com

Key words: Drug allergy; drug hypersensitivity; emergency medicine; fluid therapy; intravenous infusion.

Contribution: All authors contributed to the conception or design of the work; or the acquisition, analysis, or interpretation of data for the work, drafting the work or revising it critically for important intellectual content. All authors reviewed and approved the final version of the manuscript. Agreement to be accountable for all aspects of the work in ensuring that questions related to the accuracy or integrity of any part of the work are appropriately investigated and resolved.

Conference presentation: This study has been presented as a poster presentation at the XXVI. National Allergy and Clinical Immunology Congress between 9-13 November 2019 and EAACI Digital Congress 06 - 08 June 2020.

Conflicts of interest: The authors declare no conflict of interest.

Availability of data and materials: All data generated or analyzed during this study are included in this published article.

Ethics approval and consent to participate: The study protocol was approved by Ethics Committee. The study was conducted in accordance with the principles of the Declaration of Helsinki. All patients participating in this study signed a written informed consent form for participating in this study.

Informed consent: Written informed consent was obtained from a legally authorized representative(s) for anonymized patient information to be published in this article.

Received for publication: 11 May 2021.

Revision received: 18 July 2021.

Accepted for publication: 16 August 2021.

This work is licensed under a Creative Commons Attribution 4.0 License (by-nc 4.0).

${ }^{\circ}$ Copyright: the Author(s), 2021

Licensee PAGEPress, Italy

Emergency Care Journal 2021; 17:9848

doi:10.4081/ecj.2021.9848 to adult allergy clinic due to allergic reactions after concomitant multiple intravenous-drug administrations in Emergency Department (ED). Between January 2017 and January 2019, patients admitted to our allergy clinic with hypersensitivity reactions to intravenous drugs administered in ED were included retrospectively. Fifty-seven patients who developed allergic reactions after intravenous drug administration in EDs were evaluated. Nonsteroidal Anti-Inflammatory Drugs (NSAIDs) were the most common cause of allergic reactions $(n=40,70.2 \%)$. Skin Prick Tests (SPT) were positive in $6(10.5 \%)$ patients. Drug Provocation Tests (DPT) were positive in $10(17.5 \%)$ patients. No significant correlation was found between the total number of drugs in the intravenous fluid and the degree of allergic reaction $(r=-0.145, p$ $=0.282$ ). There was no statistically significant difference between the degree of allergic reaction and history of atopic disease $(\mathrm{p}=$ 0.579). In conclusion, concomitant administration of multiple drugs in intravenous fluids may increase the risk of allergic reactions.

\section{Introduction}

The Emergency Department (ED) is the unit where first aid is applied to emergent patients. ${ }^{1}$ Non-emergency patients also frequently apply to ED. Increases in the number of ED admissions impede the work flow of $\mathrm{ED}^{2}$ and are increasing all over the world. ${ }^{3-5}$ The increasing demand for ED is affected by the healthcare systems of the countries, the socio-demographic characteristics of the societies, and the increasing health needs. ${ }^{6,7}$ Because the recent regulations in the health-care system in Turkey, all patients presenting to ED must be admitted and examined even if their condition is not emergent. Due to these wrong health policies, patients who applied to the ED in Turkey have an expectation to have intravenous drug treatment even if they are treated orally. Therefore, drug administrations in intravenous fluids have increased in ED in Turkey and a recent study in Turkey showed that patients had a strong desire to receive intravenous treatment when admitted to the ED. ${ }^{8}$

The risk of drug allergy increases if the rate of consumption, frequency and amount of drug increase. ${ }^{9}$ Subcutaneous or intravenous drug administrations increase risk of allergic reactions. Oral drug intake has been shown to be safer than parenteral administration. It has been shown that the risk of allergic reaction increases in cases of long-term high-dose drug administration and concomitant administration of multiple drugs..$^{9-11}$ Recently, a large number of patients who developed an allergic reaction due to concomitant administration of multiple drugs in intravenous fluid in ED applied to our allergy outpatient clinic. 
The present study aimed to evaluate patients who were referred to adult allergy clinic due to allergic reactions after concomitant multiple intravenous-drug administrations in ED.

\section{Materials and Methods}

\section{Study design}

Between January 2017 and 2019, patients admitted with hypersensitivity reactions to intravenous drugs administered in ED were included retrospectively. The study protocol was approved by Ethics Committee (no: 2020/04-23). The study was conducted in accordance with the principles of the Declaration of Helsinki.

\section{Participant selection and data collection}

Demographic data, comorbidities, medical treatments in ED, degree and treatment of allergic reactions, Skin Prick Tests (SPT), Intradermal Tests (IDT) and Drug Provocation Tests (DPT) results were obtained from the medical records of the patients. The definition and severity of Type 1 systemic hypersensitivity reactions following drug infusion were determined according to the modified classification of Rueff et al. ${ }^{12}$ (Table 1).

\section{Diagnosis of drug allergy}

The diagnosis of drug allergy was based on history, physical examination findings, SPT, IDT and DPT. SPT and IDT were performed on the volar side of the forearm. Skin tests were evaluated 20 minutes after applying the culprit drug, with positive (histamine) and negative (saline) controls. Neither SPT nor IDT were performed on patients who had received antihistamines in the last seven days and who had had dermographism. In SPT, an induration diameter of $3 \mathrm{~mm}$ and over was accepted as positive. In IDT, an induration diameter of $3 \mathrm{~mm}$ or more was considered positive. DPT was performed in a single-blind manner by increasing the dose at intervals of 15-30 minutes. All DPTs were performed under the observation of an allergy specialist. DPT was not performed with culprit drugs if patients had a history of severe allergic reactions and/or anaphylaxis.

\section{Statistical analysis}

Statistical analysis was performed using SPSS 20 software (IBM). The distribution of numerical data was evaluated by Kolmogorov-Smirnov test. If numerical data were normally distributed, mean and standard deviation were used, if they were not normally distributed, the median (minimum-maximum) was used. Frequency distributions were used for categorical data. Spearman correlation analysis was used to calculate the direction and severity of the relationship between two categorical variables. Pearson chisquare test was used to evaluate the relationship between two categorical variables.

\section{Results}

Fifty-seven patients who developed allergic reactions after intravenous drug administration in EDs were evaluated. The mean age of the patients [13 $(22.8 \%)$ male, $44(77.2 \%)$ female] was $36.21 \pm 11.85$ years. The most common comorbid disease was hypertension (10.5\%). Thirty-seven $(64.9 \%)$ patients had atopic disease. The most common comorbid atopic diseases were asthma $(24.6 \%)$ and chronic urticaria and angioedema (24.6\%). The most common cause of ED admissions was upper respiratory tract infection $(56.1 \%)$. Nineteen $(33 \%)$ patients had a family history of atopy and $8(14 \%)$ patients had a family history of drug allergy. Demographic data and baseline characteristics of the patients are shown in Table 2. Nonsteroidal Anti-Inflammatory Drugs (NSAIDs) were the most common intravenously administered drugs in EDs and were the most common possible causes of allergic reactions $(n=40,70.2 \%)$. Other common intravenously administered drugs were cephalosporins $(n=13,22.8 \%)$, vitamin $B$ $(\mathrm{n}=11,19.3 \%)$, ranitidine $(\mathrm{n}=11,19.3 \%)$, metoclopramide $(\mathrm{n}=10$, $17.6 \%)$, and vitamin $\mathrm{C}(\mathrm{n}=8,14 \%)$. In terms of the drugs that cause allergic reactions, the degree and treatments are shown in Table 3.

SPT and IDT were performed on $14(24.6 \%)$ patients with at least one drug. SPT for these 14 patients were negative. IDT were positive in six $(42.8 \%)$ patients. DPT with culprit drugs were performed on $10(17.5 \%)$ patients and were positive in four $(40 \%)$ of them. The results of the diagnostic tests are shown in Table 4.

The relationship between the total number of drugs in the intravenous fluid and the degree of allergic reaction was investigated, and no significant correlation was found between the two variables $(r=-0.145, p=0.282)$. The degree of allergic reaction was compared in patients with and without a history of atopic disease. There was no statistically significant difference between the two groups $(\mathrm{p}=0.579)$.

\section{Discussion}

In the current study, in which we evaluated allergic reactions due to multiple drug infusions in serum in ED, the most frequently used drugs were NSAIDs, cephalosporins, vitamin B and ranitidine. Most of these patients could have been treated orally. A drug carries a higher risk of allergic reactions when administered intravenously than when it is administered orally; therefore, oral drug intake is safer than parenteral drug intake, ${ }^{13}$ especially in patients with a history of atopic disease and/or drug allergy. In such instances, drugs should be given orally if there is no indication for intravenous treatment. The risk of allergic reaction increases as the consumption rate, frequency and amount of the drug increase. In addition, when more than one drug is administered in the same intravenous fluid, the risk of drug allergy increases, since the risk of cross-reaction may occur. ${ }^{9}$

Table 1. Classification of severity of Type 1 systemic hypersensitivity reactions.

\begin{tabular}{ll} 
Classification & Symptom \\
Grade I & Common skin symptoms (flushing, diffuse urticaria, angioedema) \\
Grade II & Moderate-severe respiratory, cardiovascular and/or gastrointestinal symptoms \\
\hline Grade III & Anaphylactic shock, loss of consciousness \\
Grade IV & Cardiac arrest, apnea \\
\hline
\end{tabular}


In recent years, there has been an increase in ED admissions in Turkey due to an incorrect policy known as 'health transformation.' Most of these patients, who mainly had upper respiratory tract infections and/or myalgia, did not need emergency medical care and could have been treated in primary health-care centers. ${ }^{14}$ A study in Turkey showed that the most commonly diagnosed disease in EDs is upper respiratory tract disease. ${ }^{15}$ In our study population, the most common indication for intravenous treatment was also upper respiratory tract infection. NSAIDs were the most common intravenously administered drugs in EDs and were the most common causes of allergic reactions.

Multiple drug administration in intravenous fluid is also frequently observed in EDs in our country. The most important reason

Table 2. Demographic data and baseline characteristics of patients.

\begin{tabular}{|c|c|}
\hline Age (mean \pm SD) & $36.21 \pm 11.85$ \\
\hline 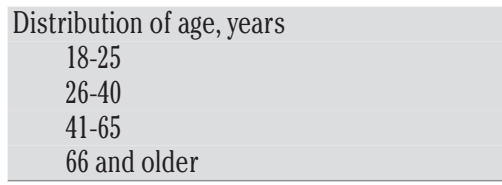 & $\begin{array}{c}\mathrm{n}(\%) \\
12(21) \\
23(40.4) \\
20(35.1) \\
2(3.5)\end{array}$ \\
\hline \multicolumn{2}{|l|}{ Gender } \\
\hline Atopic disease & $37(64.9)$ \\
\hline $\begin{array}{l}\text { Comorbiditiy } \\
\text { Asthma } \\
\text { Chronic urticaria/angioedema } \\
\text { Drug allergy } \\
\text { Allergic rhinitis } \\
\text { Hypertension } \\
\text { Diabetes mellitus } \\
\text { Goiter } \\
\text { Bee allergy } \\
\text { Cardiovascular disease } \\
\text { Chronic obstructive pulmonary disease } \\
\text { Gastroesophageal reflux } \\
\text { Family history of atopic disease } \\
\text { Family history of drug allergy }\end{array}$ & $\begin{array}{l}14(24.6) \\
14(24.6) \\
14(24.6) \\
11(19.3) \\
6(10.5) \\
4(7) \\
4(7) \\
4(7) \\
2(3.5) \\
2(3.5) \\
1(1.8) \\
19(33.3) \\
8(14)\end{array}$ \\
\hline $\begin{array}{l}\text { Reason for admission to the emergency roon } \\
\text { Upper respiratory tract infection } \\
\text { Myalgia/back pain } \\
\text { Gastrointestinal symptoms } \\
\text { (abdominal pain, diarrhea, } \\
\text { nausea, vomiting) } \\
\text { Headache } \\
\text { Poisoning }\end{array}$ & $\begin{array}{c}32(56.1) \\
10(17.6) \\
8(14)\end{array}$ \\
\hline Eozinofil count (/mm³) (median) & 3 (normal range: 0-500) \\
\hline Total IgE (IU/mL) (mean \pm SD) & 262.98 (normal range: 0-170) \\
\hline
\end{tabular}

for this is the increase in the expectations of patients from physicians to administer multiple drugs in intravenous fluid in the ED. Most of these patients could be treated with orally available drugs. Frequent and high-doses administration of multiple drugs in intravenous fluids increase the possibility of allergic reactions. ${ }^{13,16}$ The risk of cross-reactions increases when more than one drug is administered in the same intravenous fluid. In addition, as the molecular size of the drugs increases, their allergenicity increases. Due to the administration of more than one drug in the same intravenous fluid, drugs may bind to each other and haptenization of drugs may occur, which increases the allergenicity of drugs. ${ }^{9-11,16,17}$ Cross-reactions between beta-lactam antibiotics (penicillins, amoxicillin, and cephalosporins) are frequently observed in the lit-

Table 3. Culprit drugs for allergic reactions, degree and treatment of allergic reaction.

Total number of drugs in intravenous fluid

1

2

3

4

5

8

1

$21(36.8)$

$18(31.6)$

$7(12.3)$

$6(10.5)$

$4(7)$

(1.8)

Drugs administered intravenously NSAIDs

Cephalosporins

$40(70.2)$

Vitamin B

$13(22.8)$

Ranitidine

$11(19.3)$

Metoclopramide

$11(19.3)$

Vitamin C

$10(17.6)$

Hyoscine-N-butylbromide $\quad 8(14)$

Penicillin $6(10.5)$

Paracetamol $5(8.8)$

Thiocolchicoside $\quad 5(8.8)$

Proton pump inhibitor 2 (3.5)

Allergic reaction type

Type $1 \quad 56(98.2)$

Type 4

The degree of Type 1 allergic reaction

Grade 1

$22(38.6)$

Grade 2

$21(36.8)$

Grade 3

$13(22.8)$

Treatment of allergic reaction

Antihistamine

Systemic corticosteroid

$36(63.2)$

Oxygen

$32(56.1)$

Adrenali

$15(26.3)$

Beta 2 agonist

$12(22.1)$

Hospitalization

$10(17.6)$

Admission to intensive care unit

$4(7)$

Table 4. IDT and DPT results.

\begin{tabular}{lcc} 
& Result $\mathbf{n}(\%)$ & Positive Drugs n (\%) \\
IDT & Positive: $6(42.8)$ & H2 receptor blocker (ranitidine): $3(21.4)$ \\
& Negative: $8(57.2)$ & Proton pump inhibitor: $1(7.1)$ \\
& & Cephalosporins:1 $(7.1)$ \\
DPT with the culprit drug & & NSAIDs: 1 (7.1) \\
& Positive: $4(40)$ & NSAIDs: $3(30)$ \\
& Negative: $6(60)$ & Paracetamol: $1(10)$ \\
\hline
\end{tabular}


erature. ${ }^{18}$ In addition, cross-reactions between NSAIDs drug groups are frequently observed due to COX-1 (cyclooxygenase 1) inhibition. ${ }^{17}$ In our study, $63.2 \%$ of patients who developed an allergic reaction after intravenous treatment had two or more drugs in the intravenous fluid administered to them. However, in our correlation analysis, we did not find any significant connection between the number of drugs in the fluid and the severity of the allergic reaction.

In the current study, an inappropriate medical practice that we observed in the ED is the administration of drugs which are not indicated in the treatment of the patient's disease. We observed that non-indicated treatments, such as vitamins B and C, were administered intravenously to patients in EDs for upper respiratory tract infections, myalgia, back pain, and headache. This inappropriate treatment can, and did, cause allergic reactions. $19.3 \%$ of patients who developed an allergic reaction after intravenous treatment in the ED were given vitamin B, and $14 \%$ of these patients were also given vitamin $\mathrm{C}$ in intravenous fluids.

Diagnostic tests should be performed to confirm the diagnosis of drug allergy..$^{18}$ In vitro tests and valid skin tests should be performed for diagnostic purposes. When skin tests or in vitro tests are positive, this result indicates a hypersensitivity reaction. The positivity should be consistent with the clinical history of the patients. However, the sensitivity of these tests is low, and negative skin tests and in-vitro tests cannot completely exclude the diagnosis of drug allergy. Therefore, DPT, which is the gold-standard test, should be performed to confirm the diagnosis of a suspected drug allergy. ${ }^{19,20}$ DPT should be performed according to the risk-benefit ratio for each patient. In patients with a history of serious allergic reactions, DPT with alternative drugs may be performed instead of with culprit drugs. In our study, DPTs were performed with safe alternative drugs due to risk of anaphylaxis in many of patients.

This study had some limitations. We could not perform SPT and IDT with culprit drugs in many patients $(75.4 \%)$ as some drugs are not suitable for skin testing, some patients had dermographism and their previous allergic reaction was very severe, and skin tests presented a risk of anaphylaxis in others.

\section{Conclusions}

Concomitant administration of multiple drugs in intravenous fluids may increase the risk of allergic reactions. The rational use of medicine principle should be obeyed and oral treatment should be preferred if possible.

\section{References}

1. Polat O, Kabaçam G, Güler İ, et al. Surveillance analysis of the patients attended to Ibni Sina Hospital Emergency Department. Turk J Emerg Med 2005;5:78-81.

2. Ersel M, Karcıoğlu Ö, Yanturalı S, et al. Emergency Department utilization characteristics and evaluation for patient visit appropriateness from the patients' and physicians' point of view. Turk J Emerg Med 2006;6:25-35.

3. Pines JM, Pilgrim RL, Schneider SM, et al. Practical implica- tions of implementing emergency department crowding interventions: summary of a moderated panel. Acad Emerg Med 2011;18:1278-82.

4. Aboagye-Sarfo P, Mai Q, Sanfilippo FM, et al. Growth in Western Australian emergency department demand during 2007-2013 is due to people with urgent and complex care needs. Emerg Med Australas 2015;27:202-9.

5. Cowling TE, Harris MJ, Watt HC, et al. Access to general practice and visits to accident and emergency departments in England: cross-sectionalanalysis of a national patient survey. Br J Gen Pract 2014;64:e434-9.

6. He J, Hou X, Toloo S, et al. Demand for hospital emergency departments: a conceptual understanding. World J Emerg Med 2011;2:253-61.

7. Lowthian JA, Curtis AJ, Cameron PA, et al. Systematic review of trends in emergency department attendances: an Australian perspective. Emerg Med J 2011;28:373-7.

8. Tatli O, Simsek P, Gursoy A, et al. How important is intravenous fluid administration to patients presenting to the emergency department and to their families? Eurasian J Emerg Med 2018;17:65-70.

9. Gomes ER, Kuyucu S. Epidemiology and Risk Factors in Drug Hypersensitivity Reactions. Curr Treat Options Allergy 2017;4:239-57.

10. Celik GE, Karakaya G, Oztürk AB, et al. Drug allergy in tertiary care in Turkey: Results of a national survey. The ADAPT study: Adult drug allergy perception in Turkey. Allergol Immunopathol 2014;42:573-79.

11. Gamboa PM. The epidemiology of drug allergy-related consultations in Spanish Allergology services: Alergológica-2005. J Investig Allergol Clin Immunol 2009;19:45-50.

12. Rueff F, Przybilla B, Bilo MB, et al. Predictors of severe systemic anaphylactic reactions in patients with Hymenoptera venom allergy: importance of baseline serum tryptase a study of the European Academy of Allergology and Clinical Immunology Interest Group on Insect Venom Hypersensitivity. J Allergy Clin Immunol 2009;124:1047-54.

13. Adkinson, NF Jr. Risk factors for drug allergy. J Allergy Clin Immunol 1984;74:567-72.

14. Tanrıkulu CŞ, Tanrıkul Y, Karaman S. Review of the patients hospitalized from the emergency service and applying to the emergency service: a cross-sectional analysis of a training hospital. J Clin Anal Med 2014;5:128-32.

15. Kılıçaslan İ, Bozan H, Oktay C, Göksu E. Demographic properties of patients presenting to the emergency department in Turkey. Turk J Emerg Med 2005;5:5-13.

16. Demoly P, Bousquet J. Epidemiology of drug allergy. Curr Opin Allergy Clin Immunol 2001;1:305-10.

17. Warrington R, Silviu-Dan F, Wong T. Drug allergy. Allergy Asthma Clin Immunol 2018;14:60.

18. Zagursky RJ, Pichichero ME. Cross-reactivity in beta-Lactam Allergy. J Allergy Clin Immunol Pract 2018;6:72-81.e1.

19. Weiss ME, Adkinson NF. Diagnostic testing for drug hypersensitivity. Immunol Allergy Clin N Am 1998;18:731-44.

20. Aberer W, Kränke B. Provocation tests in drug hypersensitivity. Immunol Allergy Clin N Am 2009;29:567-84. 